\title{
Biocompatible Quantum Funnels for Neural Photostimulation
}

\author{
Houman Bahmani Jalali, ${ }^{\dagger}$ Onuralp Karatum, ${ }^{\ddagger \odot}$ Rustamzhon Melikov, $^{\ddagger \odot}$ Ugur Meric Dikbas, ${ }^{\S}$ \\ Sadra Sadeghi, ${ }^{\| \odot}$ Erdost Yildiz, ${ }^{\perp}$ Itir Bakis Dogru, ${ }^{\dagger}$ (๑) Guncem Ozgun Eren, ${ }^{\dagger}$ Cagla Ergun, ${ }^{\#}$ \\ Afsun Sahin, ${ }^{\perp, \nabla}$ Ibrahim Halil Kavakli, ${ }^{\S, \#}$ and Sedat Nizamoglu*, ${ }^{*}$, ,, i॰
}

${ }^{\dagger}$ Graduate School of Biomedical Science and Engineering, ${ }^{\ddagger}$ Department of Electrical and Electronics Engineering, ${ }^{\S}$ Department of Molecular Biology and Genetics, "Graduate School of Material Science and Engineering, ${ }^{\perp}$ Research Center for Translational Medicine, and ${ }^{\#}$ Department of Chemical and Biological Engineering, Koç University, Istanbul 34450, Turkey

${ }^{\nabla}$ Department of Ophthalmology, Koç University Medical School, Istanbul 34450, Turkey

\section{Supporting Information}

ABSTRACT: Neural photostimulation has high potential to understand the working principles of complex neural networks and develop novel therapeutic methods for neurological disorders. A key issue in the light-induced cell stimulation is the efficient conversion of light to bioelectrical stimuli. In photosynthetic systems developed in millions of years by nature, the absorbed energy by the photoabsorbers is transported via nonradiative energy transfer to the reaction centers. Inspired by these systems, neural interfaces based on biocompatible quantum funnels are developed that direct the photogenerated charge carriers toward the bionanojunction for effective photostimulation. Funnels are

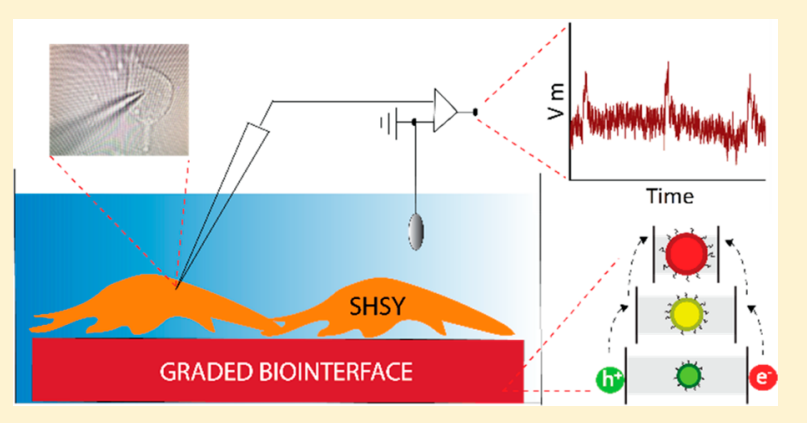
constructed with indium-based rainbow quantum dots that are assembled in a graded energy profile. Implementation of a quantum funnel enhances the generated photoelectrochemical current $215 \%$ per unit absorbance in comparison with ungraded energy profile in a wireless and free-standing mode and facilitates optical neuromodulation of a single cell. This study indicates that the control of charge transport at nanoscale can lead to unconventional and effective neural interfaces.

KEYWORDS: Biointerface, photostimulation, quantum dot, quantum funnel, indium phosphide

$\mathrm{N}$ eural stimulation technology, which constituted a \$2.7 billion market in 2017 and is anticipated to show an exponential growth reaching $\$ 11.5$ billion in 2022 in the United States, ${ }^{1}$ is expected to create opportunities for advanced treatments for a broad range of neurological disorders including retinal degeneration, hearing loss, diabetes and Alzheimer's disease. ${ }^{2}$ The conversion of light energy to neural stimulation via genetic tools, known as optogenetics, significantly advanced the communication with neural cells and tissues. ${ }^{3,4}$ Optogenetics induces photoactive ion channels via delivery of a virus to the targeted neurons and allows the modulation of membrane voltage under light illumination., 5 However, the requirement of genetic alteration of cells is considered as one of the main limitations of this technology to use it on humans. ${ }^{7}$

Alternatively, optoelectric excitation as a nongenetic approach provides high-resolution photostimulation due to their photoresponse at cellular and even subcellular levels in vivo and in vitro. ${ }^{7-10}$ In optoelectric stimulation, light is absorbed by a photoactive substrate and the substrate converts the light energy to electrical current for cell stimulation. Different types of photonic materials including organic polymers, ${ }^{11,12}$ silicon structures, ${ }^{10,13,14}$ carbon nanotubes, ${ }^{7,15}$ and semiconductor quantum $\operatorname{dots}^{16-18}$ (QDs) were used. Among these materials, QDs show wide spectral tunability ${ }^{19}$ due to quantum confinement effect and high photostability ${ }^{7}$ for the fabrication of targetable and remotely addressable biointerfaces. ${ }^{16}$ Uniquely, their photoresponse and spectral sensitivity can be controlled during QD synthesis via adjusting their size and composition ${ }^{18}$ or after-synthesis via surface engineering and assembly. ${ }^{16}$

QDs show high potency in the realm of unconventional neural interfaces. So far, most of the efforts were concentrated on the use of cadmium $(\mathrm{Cd})$ and mercury $(\mathrm{Hg})$ containing QDs. ${ }^{16,17,20}$ Release of the heavy metal ions by these QDs to the biological environment can bind mitochondrial proteins, weaken cellular respiration and lead oppoptosis. ${ }^{21}$ Moreover, in terms of the neural interface structures, layer-by-layer assembly of monosized $\mathrm{HgTe}$ QDs, ${ }^{16}$ electrostatic layer-bylayer assembly of CdTe QDs, drop-casted CdSe QDs in film forms and conjugation of $\mathrm{CdSe} / \mathrm{CdS}$ semiconductor nanorods onto carbon nanotubes were investigated. ${ }^{17,22}$ Recently, toxicheavy-metal-free $\mathrm{InP} / \mathrm{ZnO}$ type-II quantum dots were incorporated on $\mathrm{TiO}_{2}$ in a photoanode structure. ${ }^{18}$ Different from the previous studies, our concept in this study is the adaptation of nonradiative energy transfer (ET) used by

Received: April 24, 2019

Revised: $\quad$ August 8, 2019

Published: August 9, 2019 

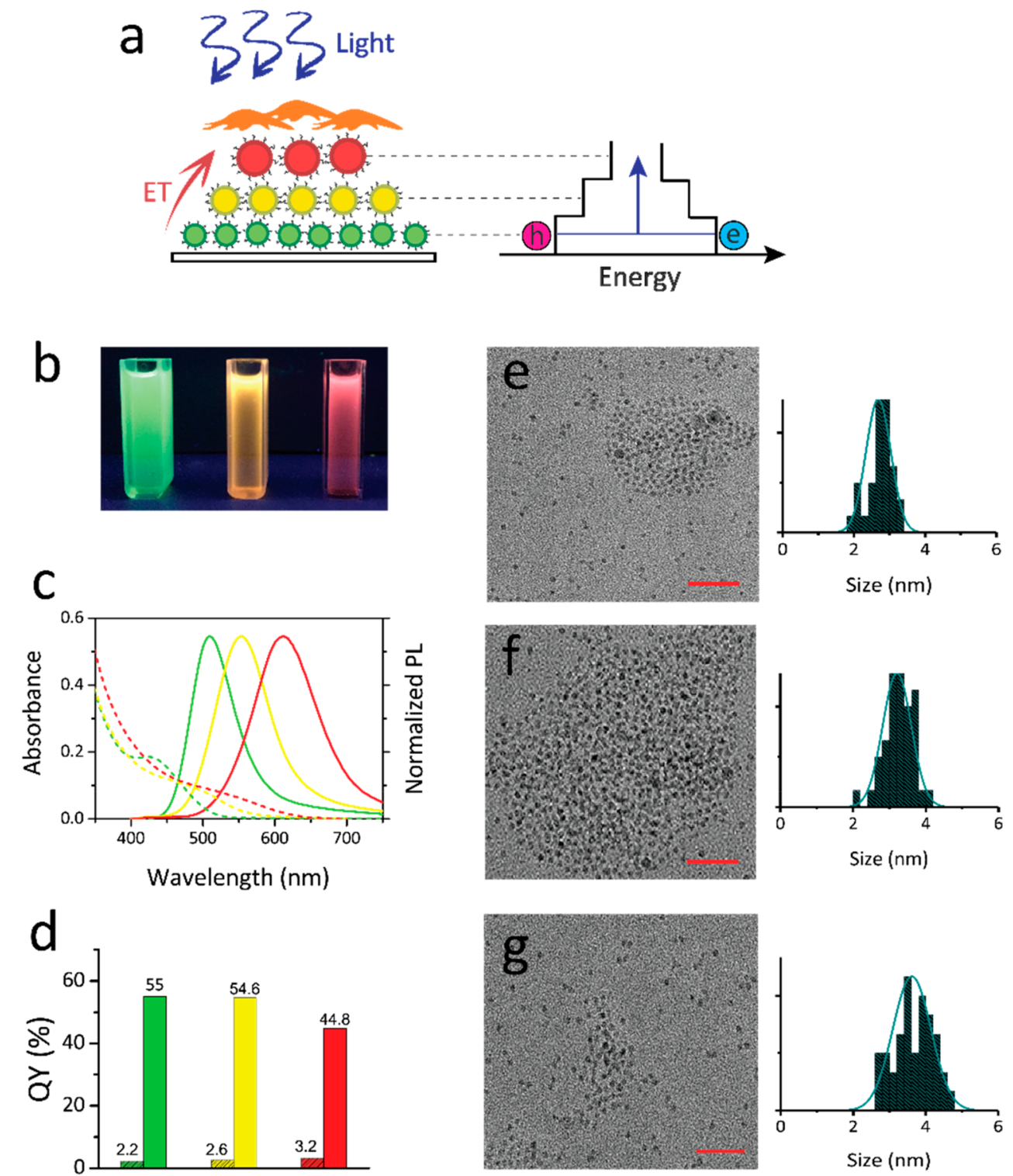

Figure 1. (a) Artifical antenna complexes made of rainbow InP quantum dots showing nonradiative energy transfer toward the cell interface. This figure is not drawn to scale. (b) Photograph of the colloidal green, yellow and red emitting QDs under UV illumination. (c) Steady-state absorption (dashed) and PL (solid) spectra of the colloidal green-, yellow-, and red-emitting QDs. (d) QY of the green-, yellow-, and red-emitting InP core and $\mathrm{InP} / \mathrm{ZnS}$ core/shell QDs. TEM and size distribution of the synthesized (e) green- $(2.68 \mathrm{~nm} \pm 0.34)$, (f) yellow- $(3.20 \mathrm{~nm} \pm 0.39)$, and $(\mathrm{g})$ red- $(3.61 \mathrm{~nm} \pm 0.52)$ emitting $\mathrm{InP} / \mathrm{ZnS}$ core/shell QDs (for the particle size distribution, minimum 30 particles were counted).

photosynthetic systems to neural interfaces. During photosynthesis, the sunlight is captured by high-energy chromophores (e.g., Chl b, phycobilins, carotenoids) and transferred to lowerenergy chromophores ( $\mathrm{Chl}$ a) in which chemical reaction centers of P680 for Photosystem II and P700 for Photosystem I are located. ${ }^{23,24}$ The mechanism of excitation energy transfer in natural antennas is based on Förster energy transfer $\left(\right.$ dipole-dipole interaction ${ }^{23}$ ). The energy transfer in the natural antenna complexes is realized via the proper energetic overlap of the molecules and the distance between neighboring molecules at nanometer levels. ${ }^{25}$ In this study, we use rainbow quantum dots as artificial antenna complexes and build a biocompatible quantum funnel for the first time that will direct the photogenerated charge carriers to the bionanojunction for neural photostimulation. In the quantum funnel structure, emission of the smaller QDs are matched with the absorption of the larger QDs, and optical excitations efficiently transfer along the band gap gradient and are finally captured by the largest QDs that will induce bioelectrical stimuli for neural photosimulation.

Results and Discussion. Construction of Quantum Funnels Using Rainbow QDs. Multilayers made of size gradient of QDs (green, yellow, and red emitting) can create quantum funnels (QFs) due to their near-field dipole-dipole coupling (Figure 1a). For that we synthesized green-, yellow-, and red-emitting InP cores by hot injection method based on our previous reports ${ }^{26,27}$ (Figure $1 \mathrm{~b}, \mathrm{c}$ ). The QY of green, yellow, and red emitting InP QDs, measured in an integrating sphere, correspond to $2.2 \%, 2.6 \%$, and $3.2 \%$, respectively (Figure 1d). Because higher QY facilitates more efficient nonradiative energy transfer due to the increase of the Förster radius according to eq $1^{28}$ (in which $\kappa^{2}, n, \Phi_{\mathrm{d}}$, and $J_{\mathrm{da}}$ are random dipole orientation factor of $2 / 3,{ }^{29}$ refractive index of the medium, QY of donor, and overlap of the donor emission 
a
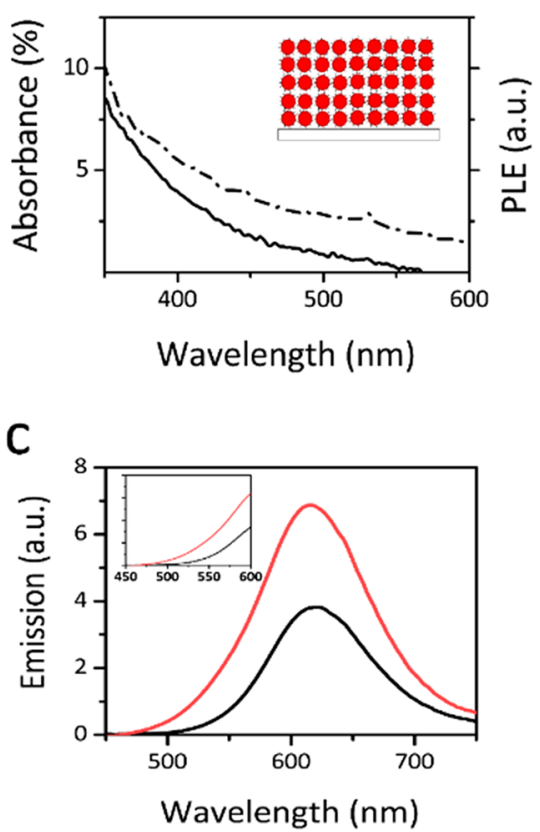

b

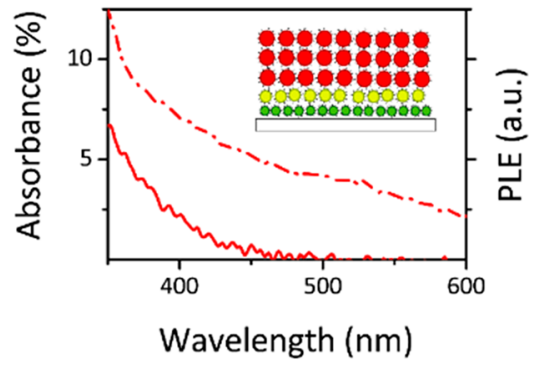

d

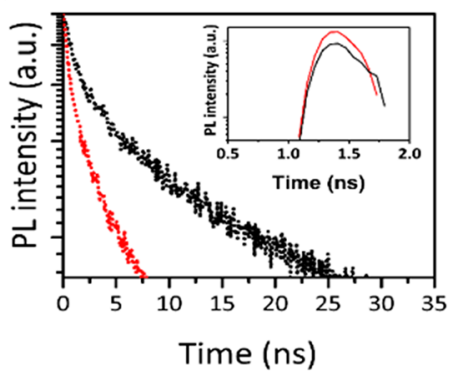

Figure 2. (a) Absorbance (solid) and PLE (dashed) spectra of the ungraded biointerface. Inset: Schematic representation of the ungraded biointerface. (b) Absorbance (solid) and PLE (dashed) spectra of the quantum funnel biointerface. Inset: Schematic representation of the graded biointerface. PLE was recorded at the emission wavelength of $620 \mathrm{~nm}$. (c) PL spectra of the ungraded (black) and quantum funnel (red) biointerfaces under the excitation wavelength of $375 \mathrm{~nm}$. Inset: Zoom-in of PL spectra of the ungraded (black) and quantum funnel (red) biointerfaces. (d) Time-resolved PL spectra of the ungraded (black) and quantum funnel (red) biointerfaces excited at $375 \mathrm{~nm}$. Inset: Instrument response function (IRF) for the analysis of ungraded (black) and quantum funnel (red) biointerfaces.

spectrum, respectively), $0.6 \mathrm{~nm} \mathrm{ZnS}$ shell $^{27}$ was coated around the InP core to increase QY of the QDs. As a result, QY of the InP core was enhanced after $\mathrm{ZnS}$ shelling by a factor of 25,21 , and 14 for green, yellow and red-emitting QDs, respectively (Figure 1d). For efficient energy transfer the steady state absorbance and PL spectra also have good spectral overlap between emission and absorbance of rainbow QDs $\left(\mathrm{J}_{\mathrm{da}}\right)$ (Figure 1c). Moreover, the transmission electron microscopy (TEM) analysis indicated an increase of a mean particle size from 2.8 to 3.2 and $3.6 \mathrm{~nm}$ for green-, yellow-, and redemitting QDs, respectively, which is in agreement with the red shift in the PL due to the quantum confinement effect (Figure $1 \mathrm{e}-\mathrm{g})$.

$$
\begin{aligned}
& R_{0}=\left(8.79 \times 10^{23}\right) \kappa^{2} n^{-4} \Phi_{\mathrm{d}} J_{\mathrm{da}} \\
& E=\frac{1}{1+\frac{R^{6}}{R_{0}^{6}}}
\end{aligned}
$$

The efficiency of nonradiative energy transfer $(E)$ is also affected by donor-acceptor and Förster distance $\left(R\right.$ and $\left.R_{0}\right)$, respectively (eq 2). ${ }^{30}$ Even though the donor-acceptor distance $(R)$ will increase after shelling, which will decrease the energy transfer efficiency by a factor of around 16 and 12 for green-yellow and yellow-red donor-acceptor pairs, the $\mathrm{QY}$ enhancement of donors is stronger to increase the energy transfer efficiency. In addition, $\mathrm{ZnS}$ shell leads to a potential barrier that will block a possible Dexter-type charge transfer that can decrease the dipole-coupling-induced energy transfer efficiency. At the same time, the QY increase after shell growth also shows the passivation of the defect states that positively affect the energy transfer of the photogenerated charge carriers without significant trapping in midgap states. Therefore, the above facts indicate that $\mathrm{ZnS}$ shelling in the biocompatible InP QD nanostructures is an appropriate strategy to form an efficient energy transfer architecture.

To facilitate energy transfer in biointerface, we constructed a quantum funnel structure made of graded energy profile using rainbow quantum dots on ITO substrate. For the construction, we used multilayer spin-coating method ${ }^{31}$ (see Experimental Section for the detailed experimental procedure). The interlayer attachment was done by using 3-mercaptopropionic acid (3-MPA) as a short $\left(6^{\circ} \mathrm{A}^{32}\right)$ bifunctional linker molecule for effective dipole-dipole coupling between donor and acceptor pairs. Because one donor QD can transfer its energy to its 3-subsequent nearby acceptor QDs, ${ }^{29}$ we deposited three layers of red-emitting QDs after the green and yellow layers. The thickness of the inorganic NPs is determined to be 14-24 $\mathrm{nm}$, which is similar in length scales with CdSe layer-by-layer assemblies. ${ }^{33}$ To compare the performance of the QF, we also fabricated a biointerface structure with an ungraded bandgap profile as a control group, made of five layers of red emitting QDs.

Energy transfer in the quantum funnel structure is observed both in steady-state and time-resolved optical characterizations. Weak photoluminescence in yellow-green spectral region and strong emission intensity in red spectral region by the QF indicates accumulation of excitons in the interfacial red layers due to energy transfer from green and yellow emitting donors to red emitting acceptors (Figure 2c). This is also observed with the stronger photoluminescence excitation (PLE) signal of graded sample (Figure 2a,b). The steadystate absorbance of ungraded biointerface is higher than the graded biointerface due to the higher extinction coefficient of 
a
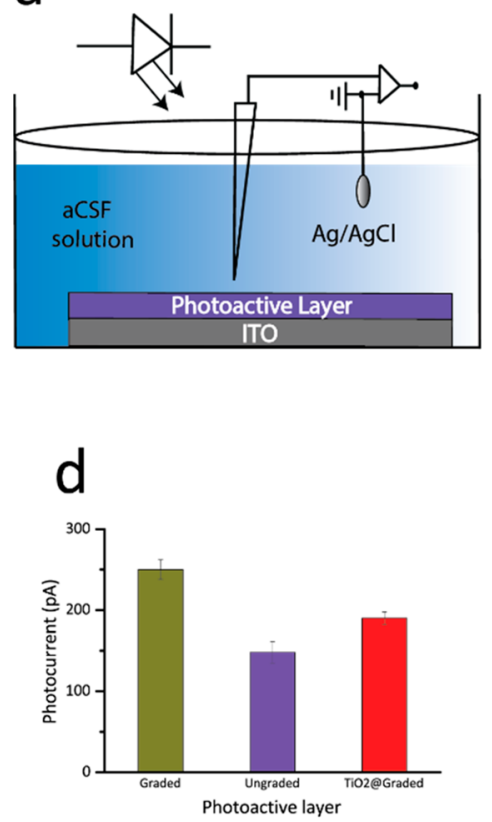

b

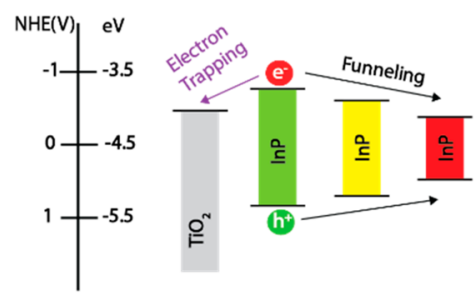

e

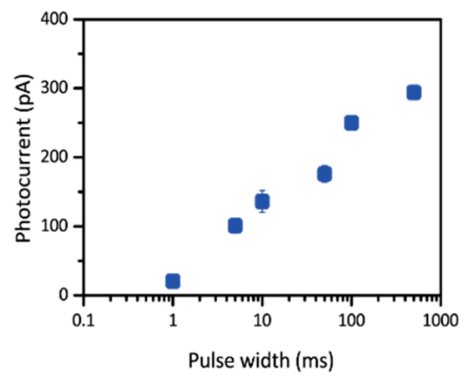

C

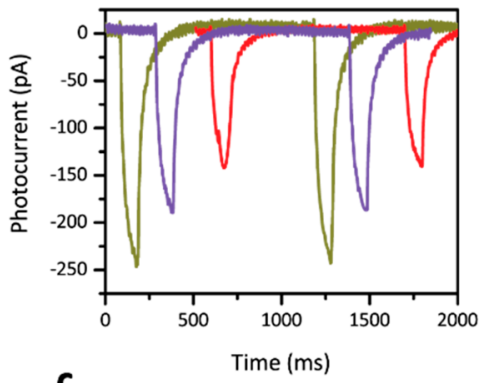

$f$

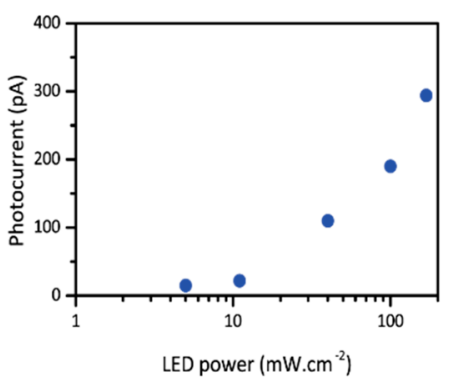

Figure 3. (a) Schematic of the photocurrent recording system. The biointerfaces are placed in wireless and free-standing mode under light illumination. (b) Band diagram of $\mathrm{TiO}_{2}$-quantum funnel biointerface. (c) Photocurrent of the ungraded (red), quantum funnel (brown), and $\mathrm{TiO}_{2}-$ quantum funnel (purple) biointerfaces under an illumination of $169 \mathrm{~mW} \cdot \mathrm{cm}^{-2}$ with the pulse duration of 100 ms. (d) Photocurrent amplitudes generated by the biointerfaces under an illumination of $169 \mathrm{~mW} \cdot \mathrm{cm}^{-2}$ with the pulse duration of $100 \mathrm{~ms}$. (e) Photocurrent generated under an illumination of $169 \mathrm{~mW} . \mathrm{cm}^{-2}$ as a function of pulse width. (f) Photocurrent generated as a function of LED optical power. The pulse duration is $500 \mathrm{~ms}$.

the red QDs compared to green QDs (Figure 2a,b). ${ }^{34}$ Hence, even though the quantum funnel biointerface has lower absorption, it can emit stronger than the ungraded one, possibly due to the recycling of the trapped excitons via energy transfer $^{35}$ (Figure 2c). We further investigated time-resolved charge carrier dynamics of the quantum funnel biointerface and for that we fabricated a donor control group made of five layers of green-emitting QDs. Biointerfaces were excited with $375 \mathrm{~nm}$ pulsed laser and their decay analyses were performed (Figure $2 \mathrm{~d}$ ). The average lifetime $\left(\tau_{\text {avg }}\right)$ was calculated from an amplitude weighted mean ${ }^{26}$ (eq 3 ). The lifetime of the greenemitting QDs in the donor control group and in the quantum funnel biointerface correspond to $6.08 \pm 0.19$ and $3.01 \pm 0.11$ ns, respectively. Here, the lifetime of the QF significantly decreased due to the effective donation of energy to the acceptor QDs (Table S1). The ET efficiency is calculated based on eq $4,{ }^{30}$ in which $\tau_{\mathrm{D}}$ and $\tau_{\mathrm{DA}}$ are the donor lifetime of the control group and of the $\mathrm{QF}$, respectively, and the ET efficiency corresponds to $51 \%$ in the funnel structure.

$$
\begin{aligned}
& \tau_{\text {avg }}=\frac{A_{1} \tau_{1}^{2}+A_{2} \tau_{2}^{2}}{A_{1} \tau_{1}+A_{2} \tau_{2}} \\
& E=1-\frac{\tau_{\mathrm{da}}}{\tau_{\mathrm{d}}}
\end{aligned}
$$

Funnel-Induced Photocurrent Enhancement. To explore the photostimulation strength of biointerfaces we investigate the photocurrent in a biological medium. An electrophysiology setup is operated and the biointerfaces are measured in the wireless and free-standing mode ${ }^{10}$ (Figure 3a). Artificial cerebrospinal fluid $(\mathrm{aCSF})$, silver/silver chloride $(\mathrm{Ag} / \mathrm{AgCl})$ electrode, and blue light-emitting diode (LED) (Figure S1) are used as the medium, reference electrode, and illumination source, respectively. The depth of the aCSF electrolyte is $1 \mathrm{~cm}$, which transmit most of the illuminated light (>95\%) (Figure $\mathrm{S} 2)$. A low drift current ( $5 \mathrm{pA})$ is observed during voltageclamp measurements due to the light sensitivity of the $\mathrm{Ag}$ / $\mathrm{AgCl}$ reference electrode.

The quantum funnel biointerface generates a mean photocurrent peak of $(0.25 \mathrm{nA} \pm 0.012)$, and the ungraded biointerface produces a mean photocurrent peak of $(0.148 \mathrm{nA}$ $\pm 0.013)$. In other words, the generated photocurrent per unit absorbance unit was enhanced from 19 to $41 \mathrm{pA}$ coming from the accumulation of photogenerated charge carriers due to quantum funneling toward the interfacial $\mathrm{QD}$ layer with the biological medium. By closing the illumination shutter, a kind of hysteresis was seen, which can be possibly due to some trapped charges within the layers. ${ }^{36,37}$ The nonspiky behavior of the photocurrent indicates a photoelectrochemical process for photocurrent generation, which can also safely trigger photostimulation of neurons. ${ }^{10}$ The origin of the photoelectrochemical current is due to the 3-mercaptopropionic acid (MPA) coating used in the fabrication of the biointerfaces, which have $\mathrm{S}^{2-}$ groups on the QDs. These groups introduce hole-capturing ability to the surface of the QDs. ${ }^{38}$ Because of this, the transferred excitons in the low-bandgap QDs facilitated significant increase in the Faradaic charge-transfer based photocurrents. The direction of the photocurrent also confirms hole movement toward the electrolyte as well.

Furthermore, we investigate the effect of charge scavenging in a quantum funnel. For that we assemble graded structure on $\mathrm{TiO}_{2}$ layer. The $\mathrm{TiO}_{2}$ layer is coated on ITO substrate by solgel dip-coating method. ${ }^{39}$ The photocurrent of this sample 
corresponds to a mean photocurrent peak of $(0.190 \mathrm{nA} \pm$ $0.008)$, which is less than quantum funnel biointerface. This shows that the removal of photogenerated charges in a quantum funnel system negatively affects movement of the excitons to the bio-nanojunction. Hence, the quantum funnel biointerface is the most efficient candidate for neural stimulation, which can generate measurable photocurrents in both short illumination pulses (Figure $3 \mathrm{e}$ ) and low excitation powers (Figure 3f).

Biocompatibility Assessment. To safely use quantum funnels for neural interfaces, they should not induce any toxic effects on cells. For evaluating the biocompatibility of the biointerfaces, mitochondrial activity and membrane integrity are measured using 3-(4,5-dimethyl-2-thiazolyl)-2,5-diphenyl tetrazolium bromide (MTT) assay and lactate dehydrogenase (LDH) assay using SH-SY5Y cells. In addition, morphology of the cells grown on biointerfaces is assessed. Results indicated that the biointerface did affect neither the cell viability nor membrane permeability of cells. Cells grown on the biointerfaces had comparable level of cell viability with respect to ITO substrate (Figure 4a), known as nontoxic material for the mammalian cells. Second, we measured LDH activities at different time points, which were statistically not significant with control substrate (Figure 4b). Moreover, we investigated the morphology of the cells grown on the biointerface and ITO
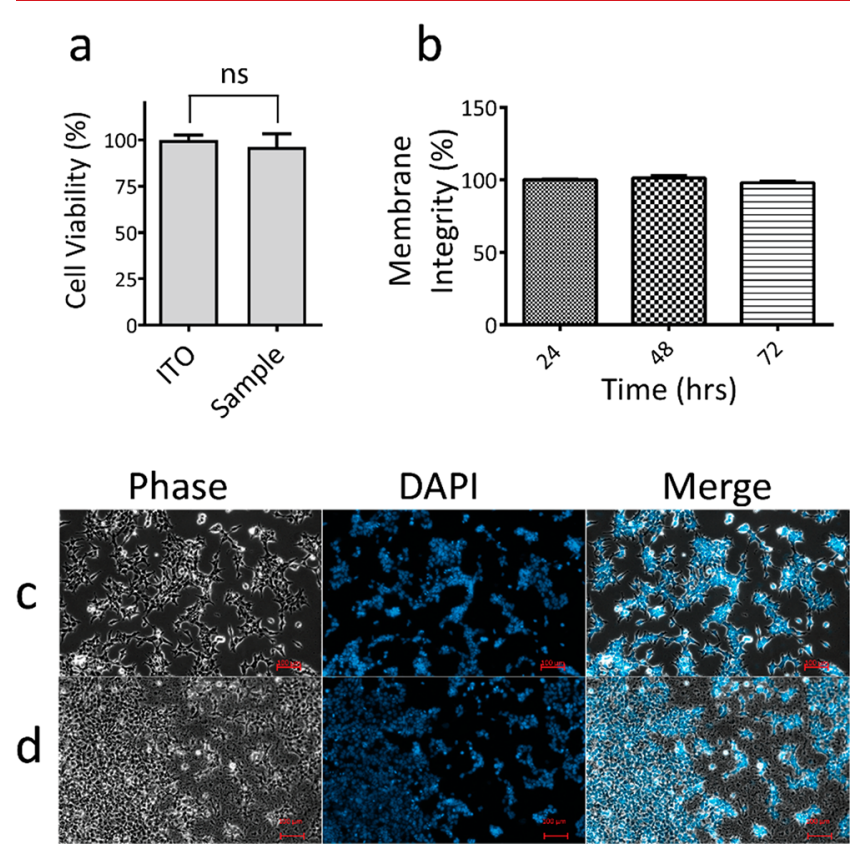

Figure 4. (a) The effect of quantum funnel biointerface on cell metabolic activity was assessed by MTT assay and compared with ITO control. An unpaired two-tailed $t$ test was performed to determine the level of significance. (b) Membrane integrity of the cells grown on biointerface was tested with LDH assay. One sample $t$ test was performed to test the significance of the difference between actual mean and theoretical mean, 100\%. Experiments were performed with at least three technical replicates and each experiment was carried out three times $(n=3)$. Results are presented in a column bar graph plotting the mean with the standard error of the mean (SEM). $p<0.05$ was considered as statistically significant and nonsignificant differences were presented as "ns". Effect of (c) ITO control and (d) the biointerface on the cell morphology visualized by fluorescence microscopy after DAPI staining and phase contrast imaging (scale bar: $100 \mu \mathrm{m}$ ). control by fluorescence microscopy, which were identical as assed by DAPI staining and phase contrast imaging (Figure 4c,d).

Funnel-Induced Neural Photostimulation. For electrophysiology measurements, SH-SY5Y cell lines are grown on the quantum funnel biointerface and we use the patch-clamp technique (Figure 5a) in the whole cell recording config-
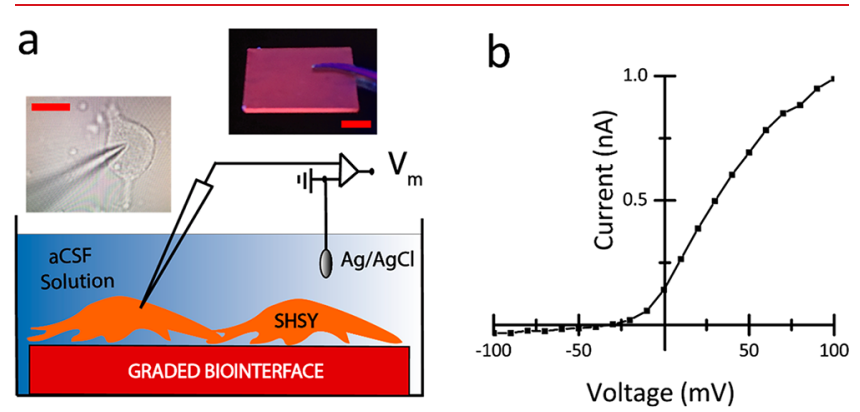

C

d
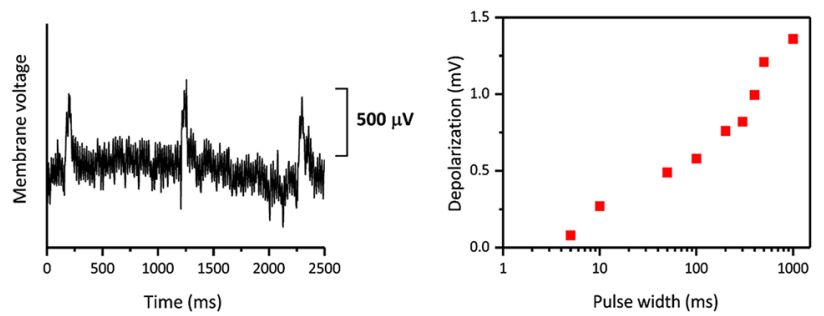

Figure 5. (a) Schematic of electrophysiology setup. Left inset: Image of the patch-clamped SH-SY5Y cell; scale bar, $10 \mu \mathrm{m}$. Right inset: Photograph of the graded biointerface under UV illumination; scale bar, $5 \mathrm{~mm}$. (b) $I-V$ curve of a SH-SY5Y cell. (c) Photostimulation of SH-SY5Y cell on the quantum funnel biointerface under illumination of $169 \mathrm{~mW} \cdot \mathrm{cm}^{-2}$ with $50 \mathrm{~ms}$ illumination pulses. (d) The membrane depolarization under different pulse durations.

uration. The biointerfaces are placed in wireless and freestanding mode. Figure $5 \mathrm{~b}$ shows a typical $I-V$ curve of the $\mathrm{SH}$ SY5Y cell line on the photoactive electrode. For photoactivation, we illuminate the biointerface at the wavelength of $450 \mathrm{~nm}$ with different pulse widths. Because the photocurrent direction is from biointerface to the electrolyte in the attached membrane, we expect to observe a membrane depolarization in the free membrane under illumination (Figure 3c). We apply $50 \mathrm{~ms}$ light pulses and observe membrane depolarization of $490 \mu \mathrm{V} \pm 17$ on SH-SY5Y cells. We further increased the pulse width of light pulses to 100 and $500 \mathrm{~ms}$, and the depolarization level corresponds to $580 \mu \mathrm{V} \pm 23$ and $1.21 \mathrm{mV} \pm 0.03$, respectively (Figure $3 \mathrm{~d}$ ). The photoelectrochemical modulation is also due to the high biocompatibility of the biointerface that leads to a reliable bionanojunction. In addition, no interfacial layer was used to enhance the biocompatibility and to promote the cell adhesion on the biointerface that may decrease the effectiveness of the cell stimulation. The quantum funnel approach presents a new tool for biocompatible and effective QD-based biointerfaces, in which improved photocurrent levels have the potential to elicit action potentials both in vitro and in vivo. ${ }^{14}$ They can be used for biological and medical applications requiring light-activated control of the neural activity and neuro-related diseases such as retinal degeneration due to retinitis pigmentosa. Because the biointerface is based on Faradaic charge-transfer mechanism 
for photostimulation, the byproducts of the reactions need to be well controlled and kept at appropriate levels that can be tolerated by the physiological buffering systems for long-term in vivo operation. 40

In summary, biointerfaces based on biocompatible quantum funnels are demonstrated for the first time. Like in photosynthetic systems, the quantum funnels convert the light energy to photogenerated charge carriers and bring the collected energy to the bionanointerface for effective photoelectrochemical modulation of neurons. We constructed the quantum funnels made of size gradient InP QDs and they show a high energy transfer efficiency of $51 \%$. In comparison with the control groups, quantum funnel biointerfaces generate 2.15-fold higher photocurrent per unit absorbance. Hence, the neural interfaces using quantum funnels can induce transmembrane potential variations on a single neuron in a wireless and free-standing mode. These findings point out that nanoengineering of QD-based biointerfaces hold great promise for unconventional neural interfaces.

Experimental Section. Rainbow QD Synthesis. InP/ZnS QDs were synthesized by Nann's method. ${ }^{28}$ To the synthesis of green-emitting InP cores, first, $0.1 \mathrm{mmol}$ of indium chloride $\left(\mathrm{InCl}_{3}\right)$, hexadecylamine (HDA), stearic acid (SA) and $3 \mathrm{~mL}$ of 1-octadecene (ODE) were mixed in a nitrogen $\left(\mathrm{N}_{2}\right)$ glovebox. The solution was heated up to $120{ }^{\circ} \mathrm{C}$ and repeatedly evacuated and refilled with nitrogen to obtain oxygen and moisture free environment. Then the flask was heated up to $220{ }^{\circ} \mathrm{C}$ and $0.5 \mathrm{~mL}$ of tris(trimethylsilyl)phosphine solution $\left(\mathrm{P}(\mathrm{TMS})_{3}\right)\left(0.2 \mathrm{mmol} \cdot \mathrm{ml}^{-1}\right.$ in ODE) was injected to the flask. The temperature was decreased to $200{ }^{\circ} \mathrm{C}$ and kept for $20 \mathrm{~min}$. Afterward, the flask was cooled down to room temperature. For $\mathrm{ZnS}$ shelling, $0.15 \mathrm{mmol}$ of zincdithiolcarbomate and $2 \mathrm{~mL}$ of ODE were added to the $\mathrm{InP}$ core solution and heated up to the $200{ }^{\circ} \mathrm{C}$ for $20 \mathrm{~min}$ to $\mathrm{ZnS}$ shell growth. Finally, the flask was cooled down to $50{ }^{\circ} \mathrm{C}$ and $4 \mathrm{~mL}$ of toluene was added and centrifuged in $4000 \mathrm{rpm}$ for $5 \mathrm{~min}$. The clear supernant solution was discarded. The QDs were separated by adding ethanol/acetone followed by centrifuging in $8000 \mathrm{rpm}$ for $15 \mathrm{~min}$. The final product was dispersed in toluene and stored in $-4{ }^{\circ} \mathrm{C}$. For yellow- and redemitting $\mathrm{QDs}$, the $\mathrm{P}(\mathrm{TMS})_{3}$ injection temperature was 260 and $280{ }^{\circ} \mathrm{C}$, respectively.

Titanium Dioxide Layer. Titanium dioxide layer was coated on ITO substrate by sol-gel dip-coating method as reported previously. ${ }^{39,41}$

Fabrication of Biointerfaces. Precleaned indium-doped tin oxide glasses $(20 \mathrm{~mm} \times 15 \mathrm{~mm}$, TEC8 Ossila) were activated by UV-ozone treatment to enhance the performance of the biointerfaces. $0.1 \mathrm{wt} \%$ 3-mercaptopropionic acid (3-MPA) in methanol was deposited by spin-coating. Afterward, QDs layers and 3-MPA as the linker were spin-coated at $5000 \mathrm{rpm}$.

Cell Culture. The electrophysiology and biocompatibility experiments were performed with SH-SY5Y, a neuroblastoma cell line. The growth medium was prepared using Dulbecco's Modified Eagle's Medium (DMEM, Gibco 41966-029) with $10 \%$ heat-inactivated fetal bovine serum (FBS, Gibco 10500) and antibiotics (1\% penicillin-streptomycin, Gibco 15240062 ). The cells were cultured at in $85 \%$ humidified incubator at $37{ }^{\circ} \mathrm{C}$ with $5 \% \mathrm{CO}_{2}$. To subculture the cells every 3 days, Trypsin/EDTA (0.25\%:0.02\%, PAN Biotech) was used for the detachment of cells from the culture plate.

Cell Viability Assay. MTT was purchased from AppliChem, Germany. MTT cell viability assay was used for evaluating biocompatibility of the materials. The materials were sterilized first by cleaning with $70 \%$ ethanol followed by air-drying. Their surface was further sterilized with UV irradiation for $30 \mathrm{~min}$. The substrates were placed in 6-well plates. SH-SY5Y cells were seeded $(3 \times 106$ cells per well $)$ on the substrates. SHSY5Y cells were grown in DMEM with $10 \%$ FBS. After $48 \mathrm{~h}$ of incubation, the medium was replaced with $1 \mathrm{~mL}$ of MTT solution $(5 \mathrm{mg} / \mathrm{mL}$ in PBS, $\mathrm{pH}=7.4)$ and $4 \mathrm{~mL}$ of DMEM mixture per well. For an additional $4 \mathrm{~h}$, the cells were incubated at $37{ }^{\circ} \mathrm{C}$ and $5 \% \mathrm{CO}_{2}$ atmosphere. Then, the medium was vacuumed from each well and substrates were transferred to an empty 6-well plate. In each well, 1:1 mixture of DMSO and ethanol was added to dissolve the formazan crystals. The solution was transferred to a 96-well plate and the absorbance was measured at $600 \mathrm{~nm}$ with Synergy H1Microplate Reader (Bio-Tek Instruments).

Fluorescence Microscopy. SH-SY5Y cells were seeded on ITO control substrate and biointerface. After $48 \mathrm{~h}$ incubation at $37^{\circ} \mathrm{C}$, cells were fixed by $4 \%$ paraformaldehyde and washed three times with PBS. The samples were blocked with PBS-bt (phosphate buffered saline, $0.1 \%$ Tween 20, and 5\% bovine serum albumin (BSA)) and subsequently stained with DAPI. Substrates were mounted by Mowiol and imaged on a flouresence microscope (Zeiss-ObserverZ1) using Zen 2 blue edition software (Zeiss).

Absorbance and Photoluminesence. Steady-state absorbance and PL were measured using an Edinburgh Instruments FL-50 spectrofluorometer by excitation wavelength of $375 \mathrm{~nm}$. The excitation and emission bandwidths were set as 2 and 3 $\mathrm{nm}$, respectively.

Time-Resolved Photoluminescence. Fluorescence decay was taken with a PicoQuant MicroTime 100 time-resolved confocal fluorescence microscope. The excitation beam was provided by $8 \mathrm{~mW}$ picosecond diode laser heads operating at $375 \mathrm{~nm}$ pulsed at $20 \mathrm{MHz}$ repetition rate.

Photocurrent Measurement. The photocurrent produced by the biointerface was measured in freestanding and wireless mode with electrophysiology system amplifier (EPC800 system made by HEKA) operated in voltage clamp mode with a patch pipet resistance of 6-8 $\mathrm{M} \Omega$ positioned closer than $10 \mu \mathrm{m}$ to the electrode surface, A Thorlabs LED (M450LP1) (450 nm, $\left.169 \mathrm{~mW} / \mathrm{cm}^{2}\right)$ served as a pulsed illumination source (LED's spectrum in Figure S1), and artificial cerebrospinal fluid (aCSF) was used as an electrolytic solution which has around $>95 \%$ transmittance in the visible range (Figure S2). Photocurrent of three different samples at three different points were measured to report average and error bar values.

Electrophysiology Test. An EPC800 system made by HEKA was used for electrophysiology experiments. SHSY-5Y neural cells were seeded on biointerface. Voltage and current of cell membrane were recorded with capillary pipettes $(6-8 \mathrm{M} \Omega)$ at room temperature under whole cell recording. Capillary pipettes contained a standard internal solution $140 \mathrm{mM}$ $\mathrm{NaCl}, 10 \mathrm{mM} \mathrm{KCl}, 5 \mathrm{mM} \mathrm{MgCl}, 10 \mathrm{mM}$ HEPES, $0.5 \mathrm{mM}$ EGTA, and $0.5 \mathrm{mM}$ ATP, with $\mathrm{pH}$ adjusted using $\mathrm{KOH}$ to 7.2. The standard external solution contained $128 \mathrm{mM} \mathrm{NaCl}, 5$ $\mathrm{mM} \mathrm{KCl}, 1 \mathrm{mM} \mathrm{MgCl}_{2}, 2.5 \mathrm{mM} \mathrm{CaCl}_{2}$, and $\mathrm{pH}$ adjusted to 7.4 with $\mathrm{NaOH}$. The same blue LED $\left(450 \mathrm{~nm}, 169 \mathrm{~mW} / \mathrm{cm}^{2}\right)$ was used as pulsed illumination source (LED's spectrum in Figure S1). 


\section{ASSOCIATED CONTENT}

\section{S Supporting Information}

The Supporting Information is available free of charge on the ACS Publications website at DOI: 10.1021/acs.nanolett.9b01697.

LED's spectrum, transmittance of aCSF electrolyte solution, and PL decay analysis data (PDF)

\section{AUTHOR INFORMATION}

Corresponding Author

*E-mail: snizamoglu@ku.edu.tr.

ORCID $\odot$

Houman Bahmani Jalali: 0000-0001-7212-9098

Onuralp Karatum: 0000-0002-7669-9589

Rustamzhon Melikov: 0000-0003-2214-7604

Sadra Sadeghi: 0000-0002-8569-1626

Erdost Yildiz: 0000-0001-8086-3524

Itir Bakis Dogru: 0000-0001-8569-7625

Sedat Nizamoglu: 0000-0003-0394-5790

Notes

The authors declare no competing financial interest.

\section{ACKNOWLEDGMENTS}

This project has received funding from the European Research Council (ERC) under the European Union Horizon 2020 Research and Innovation Programme (Grant Agreement 639846). S.N. acknowledges the support by the Turkish Academy of Sciences (TÜBA-GEBIP; The Young Scientist Award Program) and the Science Academy of Turkey (BAGEP; The Young Scientist Award Program). The authors gratefully acknowledge use of the services and facilities of the Koç University Research Center for Translational Medicine (KUTTAM), funded by the Republic of Turkey Ministry of Development. The content is solely the responsibility of the authors and does not necessarily represent the official views of the Ministry of Development.

\section{REFERENCES}

(1) Krames, E.; Peckham, P. H.; Rezai, A. R. Neuromodulation: Comprehensive Textbook of Principles, Technologies, and Therapies; Academic Press, 2018.

(2) Laxton, A. W.; Tang-Wai, D. F.; McAndrews, M. P.; Zumsteg, D.; Wennberg, R.; Keren, R.; Wherrett, J.; Naglie, G.; Hamani, C.; Smith, G. S.; et al. Ann. Neurol. 2010, 68 (4), 521-534.

(3) Zhang, F.; Gradinaru, V.; Adamantidis, A. R.; Durand, R.; Airan, R. D.; De Lecea, L.; Deisseroth, K. Nat. Protoc. 2010, 5 (3), 439.

(4) Claridge-Chang, A.; Roorda, R. D.; Vrontou, E.; Sjulson, L.; Li, H.; Hirsh, J.; Miesenböck, G. Cell 2009, 139 (2), 405-415.

(5) Lagali, P. S.; Balya, D.; Awatramani, G. B.; Münch, T. A.; Kim, D. S.; Busskamp, V.; Cepko, C. L.; Roska, B. Nat. Neurosci. 2008, 11 (6), 667

(6) Lima, S. Q.; Miesenböck, G. Cell 2005, 121 (1), 141-152.

(7) Bareket-Keren, L.; Hanein, Y. Int. J. Nanomed. 2014, 9 (1), 6583.

(8) Samia, A. C. S.; Dayal, S.; Burda, C. Photochem. Photobiol. 2006, 82 (3), 617-625.

(9) Zrenner, E.; Bartz-Schmidt, K. U.; Benav, H.; Besch, D.; Bruckmann, A.; Gabel, V.-P.; Gekeler, F.; Greppmaier, U.; Harscher, A.; Kibbel, S.; et al. Proc. R. Soc. London, Ser. B 2011, 278 (1711), 1489-1497.

(10) Parameswaran, R.; Carvalho-de-Souza, J. L.; Jiang, Y.; Burke, M. J.; Zimmerman, J. F.; Koehler, K.; Phillips, A. W.; Yi, J.; Adams, E. J.; Bezanilla, F.; et al. Nat. Nanotechnol. 2018, 13 (3), 260.
(11) Ghezzi, D.; Antognazza, M. R.; Dal Maschio, M.; Lanzarini, E.; Benfenati, F.; Lanzani, G. Nat. Commun. 2011, 2, 166.

(12) Ghezzi, D.; Antognazza, M. R.; Maccarone, R.; Bellani, S.; Lanzarini, E.; Martino, N.; Mete, M.; Pertile, G.; Bisti, S.; Lanzani, G.; et al. Nat. Photonics 2013, 7 (5), 400-406.

(13) Suzurikawa, J.; Takahashi, H.; Kanzaki, R.; Nakao, M.; Takayama, Y.; Jimbo, Y. Appl. Phys. Lett. 2007, 90 (9), 093901.

(14) Jiang, Y.; Li, X.; Liu, B.; Yi, J.; Fang, Y.; Shi, F.; Gao, X.; Sudzilovsky, E.; Parameswaran, R.; Koehler, K.; et al. Nat. Biomed. Eng. 2018, 2 (7), 508.

(15) Bareket-Keren, L.; Hanein, Y. Front. Neural Circuits 2013, 6, 122

(16) Pappas, T. C.; Wickramanyake, W. S.; Jan, E.; Motamedi, M.; Brodwick, M.; Kotov, N. A. Nano Lett. 2007, 7 (2), 513-519.

(17) Lugo, K.; Miao, X.; Rieke, F.; Lin, L. Y. Biomed. Opt. Express 2012, 3 (3), 447-454.

(18) Bahmani Jalali, H.; Mohammadi Aria, M.; Dikbas, U. M.; Sadeghi, S.; Ganesh Kumar, B.; Sahin, M.; Kavakli, I. H.; Ow-Yang, C. W.; Nizamoglu, S. ACS Nano 2018, 12, 8104.

(19) Alivisatos, A. P. J. Phys. Chem. 1996, 100 (31), 13226-13239.

(20) Molokanova, E.; Bartel, J.; Zhao, W.; Naasani, I.; Ignatius, M.; Treadway, J.; Savtchenko, A. Biophotonics Int. 2008, 15 (6), 26.

(21) Derfus, A. M.; Chan, W. C.; Bhatia, S. N. Nano Lett. 2004, 4 (1), 11-18.

(22) Bareket, L.; Waiskopf, N.; Rand, D.; Lubin, G.; David-Pur, M.; Ben-Dov, J.; Roy, S.; Eleftheriou, C.; Sernagor, E.; Cheshnovsky, O.; et al. Nano Lett. 2014, 14 (11), 6685-6692.

(23) Mirkovic, T.; Ostroumov, E. E.; Anna, J. M.; van Grondelle, R.; Govindjee; Scholes, G. D. Chem. Rev. 2017, 117 (2), 249-293.

(24) Govindjee, R.; et al. Sci. Am. 1974, 231 (6), 68-82.

(25) van Grondelle, R.; Novoderezhkin, V. I. Phys. Chem. Chem. Phys. 2006, 8 (7), 793-807.

(26) Bahmani Jalali, H.; Melikov, R.; Sadeghi, S.; Nizamoglu, S. J. Phys. Chem. C 2018, 122 (22), 11616-11622.

(27) Kumar, B. G.; Sadeghi, S.; Melikov, R.; Aria, M. M.; Jalali, H. B.; Ow-Yang, C. W; Nizamoglu, S. Nanotechnology 2018, 29 (34), 345605.

(28) Dos Remedios, C. G.; Moens, P. D. J. Struct. Biol. 1995, 115 (2), 175-185.

(29) Achermann, M.; Petruska, M. A.; Crooker, S. A.; Klimov, V. I. J. Phys. Chem. B 2003, 107 (50), 13782-13787.

(30) Förster, T. Ann. Phys. 1948, 437, 55-75.

(31) Kramer, I. J.; Levina, L.; Debnath, R.; Zhitomirsky, D.; Sargent, E. H. Nano Lett. 2011, 11 (9), 3701-3706.

(32) Narayanan, S. S.; Sinha, S. S.; Verma, P. K.; Pal, S. K. Chem. Phys. Lett. 2008, 463 (1-3), 160-165.

(33) Jin, F.; Zheng, M.-L.; Liu, Z.-H.; Fan, Y.-M.; Xu, K.; Zhao, Z.S.; Duan, X.-M. RSC Adv. 2016, 6 (30), 25401-25408.

(34) Ministro, J. A study on the synthesis and the optical properties of InP-based quantum dots. M. Sc. Thesis, University of Ghent, Belgium, 2013.

(35) Franzl, T.; Klar, T. A.; Schietinger, S.; Rogach, A. L.; Feldmann, J. Nano Lett. 2004, 4 (9), 1599-1603.

(36) Ruland, A.; Schulz-Drost, C.; Sgobba, V.; Guldi, D. M. Adv. Mater. 2011, 23 (39), 4573-4577.

(37) Tackeuchi, A.; Miyata, S.; Sugawa, S.; Kusunoki, K.; Kim, T. W.; Kim, J.-H.; Lee, H. S.; Park, H. L. Appl. Phys. Lett. 2006, 89 (11), 112125 .

(38) Wang, J. J.; Li, Z. J.; Li, X. B.; Fan, X. B.; Meng, Q. Y.; Yu, S.; Li, C. B.; Li, J. X.; Tung, C. H.; Wu, L. Z. ChemSusChem 2014, 7 (5), $1468-1475$.

(39) Jalali, H.; Trabzon, L. Materialwiss. Werkstofftech. 2016, 47 (7), 657-664.

(40) Merrill, D. R.; Bikson, M.; Jefferys, J. G. J. Neurosci. Methods 2005, 141 (2), 171-198.

(41) Jalali, H.; Trabzon, L.; Kizil, H. Aust. J. Basic Appl. Sci. 2014, 8, 259-263. 